\section{Expression of matrix metalloproteinases of human lens epithelial cells in the cultured lens capsule bag}

\begin{abstract}
Aim of purpose To observe the different expression of matrix metalloproteinases (MMPs) between pre- and postoperation of sham cataract surgery in vitro human lens capsule bag model from the same donor eye in order to investigate a possible role of MMPs in posterior capsule opacification (PCO). Methods Sham cataract surgeries were performed in six human donor eyes.

Immunohistochemical staining was used to detect the expression of MMP-2 and -9 of human lens epithelial cells (LECs) on the anterior capsulorhexis. LEC migration on posterior capsule of human lens from the same donor eye was observed in a modified capsule bag model without pin. Total MMP-2 and -9 protein production were determined by enzyme-linked immunosorbent assay at days $2,10,20$, and 30 postoperation, respectively. Results MMP-2 and -9 could not be detected immunohistochemically on the anterior capsulorhexis preoperation of cataract. Lens epithelia cells at the equator began to migrate by day 4 . A confluent monolayer of lens epithelia cells was present on the posterior capsule at day 20 . Total MMP-2 and -9 protein production increased with time with maximum levels reached on day 30 .

Conclusion MMP-2 and -9 were showed to be upregulated following sham cataract surgery. MMP expression could play an important role in PCO.

Eye (2008) 22, 439-444; doi:10.1038/sj.eye.6702735; published online 16 February 2007
\end{abstract}

Keywords: lens epithelial cells; matrix metalloproteinases; posterior capsule opacification

JH Li', NL Wang ${ }^{1}$ and JJ Wang ${ }^{2}$

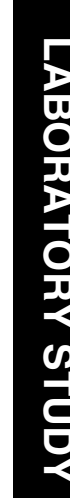

Introduction

Posterior capsule opacification (PCO) is the major cause of secondary visual loss following cataract surgery. The proliferation and migration of lens epithelial cells (LECs) at the equator and under the anterior lens capsule following cataract surgery lead to the development of PCO. Why do lens epithelia cells remain quiet in closed capsule bag but become active in open environment following cataract surgery?

Matrix metalloproteinases (MMPs) are a group of proteolytic enzymes which are important in many physiological processes such as embryogenesis, development, and wound healing. ${ }^{1}$ They have often been shown to be upregulated during wound healing. ${ }^{2}$ MMPs help cells to penetrate and move through the extracellular matrix (ECM) and basement membrane by cleaning components of the ECM.

The main component of lens posterior capsule is callagen type IV and laminin which are the substrates of MMP-2 and -9. ${ }^{3}$ The development of PCO resulting from lens epithelia cell growth is essentially a wound healing response.

Therefore, we hypothesis that wound healing response following cataract surgery induces the secretion of MMP-2 and -9 in residual lens epithelia cells. MMPs improve the lens epithelia cells at the equator to migrate onto the posterior capsule. These cells then induce matrix modification such as wrinkling and cell aggregation, which results in PCO. The objectives of this study were to observe the different expression between pre- and postoperation of sham cataract surgery in the same donor eye in order to investigate a possible role of MMPs in PCO. These aims were achieved by immunohistochemistry staining and enzyme-linked immunosorbent assay
${ }^{1}$ Beijing Tongren Eye Center, Beijing Tongren Hospital Affiliated Hospital of Capital Medical University, Beijing, China

${ }^{2}$ Beijing Institute of Ophthalmology, Beijing, China

Correspondence: NL Wang, Beijing Tongren Eye Center, Beijing Tongren Hospital, Affiliated Hospital of Capital Medical University, No. 1 Dongjiaomingxiang, Dongchen district, Beijing, 100730, PR China

Tel: + 86010 58269920;

Fax: + 8601058269920

E-mail: wningli@

trhos.com

Received: 30 June 2006 Accepted in revised form: 28 December 2006 Published online: 16 February 2007 
(ELISA) analysis of production of cultured human lens capsular bags generated by a sham cataract operation.

\section{Materials and methods}

\section{Retrieval and preparation of human lens capsule bags}

Six donated human eyes were obtained from Tongren Eye Bank (Beijing, China). The research followed the tenets of the Declaration of Helsinki regarding the use of human material. The mean (standard deviation) ages of the donors were 48(SD 5) years for four male donors and 50(SD 2.8) years for two female donors. Lenses used in the study (total six) were retrieved within $12 \mathrm{~h}$ postmortem. Lens capsule bag culture was established using a technique described previously by Liu et al. ${ }^{4}$ But no pin was used to fix the capsule bag. Briefly, a sham cataract operation was performed, which involved anterior capsulorhexis for $5 \mathrm{~mm}$ in diameter, nuclear hydroexpression with phosphate-buffered saline (PBS), and the aspiration of cortical lens fibres. The capsular bag was then dissected free of the zonules and secured on a six-well culture plate. The lens capsular bag was maintained in $1.5 \mathrm{ml}$ nonsupplemented Eagle's minimum essential medium (Sigma, Poole, UK). The capsule bag was opened by shaking the culture plate gently as soon as the bag was transferred into the culture media. The capsule bag in culture media retained circular shape and was full of media all the time. No pin was used to fix the capsule bag. The capsule bag changed its position in the media when the culture plate was moved. The specimens were incubated at $37^{\circ} \mathrm{C}$ in $5 \%$ humidified $\mathrm{CO}_{2}$ in air. Ongoing observations were performed using phase-contrast microscopy.

\section{Immunohistochemistry}

The anterior capsulorhexis was subjected to immunohistochemistry staining. Immunohistochemical assays were performed on formalin-fixed and paraffin-embedded tissues with the SP kit (Beijing Zhongshan Company, Beijing, China). In brief, the paraffin blocks were sliced into $4 \mu \mathrm{m}$-thick sections. Sections were deparaffinized in xylene for $24 \mathrm{~h}$, dehydrated with graded ethanol washes (100-70\%), and then incubated with hydrogen peroxide and protein block for $10 \mathrm{~min}$ each to block endogenous peroxidase activity. Sections were subsequently washed in PBS buffer. Mouse monoclonal antibodies against human MMP-2 and -9 (1:500) were reacted, respectively with tissue specimens at $37^{\circ} \mathrm{C}$ for $1 \mathrm{~h}$, followed by sequential incubations with biotin-goat anti-mouse $\operatorname{IgG}(1: 200)$ at $37^{\circ} \mathrm{C}$ for $15 \mathrm{~min}$, streptavidin-peroxidase $(1: 200)$ conjugated avidin reagent, and amplification reagent for at $37^{\circ} \mathrm{C}$ for $15 \mathrm{~min}$ each. Diaminobenzidine was used as the chromogen, and sections were counterstained with haematoxylin. For the negative control, the primary antibody was replaced with PBS. Human ciliary smooth muscles were obtained at the same time and used as positive controls. ${ }^{5}$

\section{MMP protein production}

The media were sampled and replaced daily. The culture media bathing the lens capsules were collected on days 2, 10,20 , and 30 ; stored at $-70^{\circ} \mathrm{C}$ then lyophilized and reconstituted in PBS before use. Total MMP-2 and -9 was quantified in the collected culture media taken from the epithelial cell populated lens capsules by ELISA. The ELISA kits (Rapidbio, USA) were solid phase enzyme immunoassays based on a two-step sandwich format and measured both the latent and active forms of the enzymes. A test run was performed for each assay to determine the appropriate dilution factor of $100 \mu \mathrm{l}$ of each collected sample culture medium to ensure that the readings correlated with the exponential part of the respective standard curves. To eliminate the possibility of interassay variation for each ELISA, the samples were assayed in one run. The assays were performed in accordance with the manufacturer's instructions.

\section{Statistical evaluation}

Statistical graphs were performed using SPSS11.5.

\section{Results}

\section{Immunohistochemistry}

Light micrographs of the immunostained specimens revealed MMP-2 (Figure 1a) and MMP-9 (Figure 1b) localized to the cytoplasm in a fine granular pattern in human ciliary smooth muscle cells. MMP-2 (Figure 1c) and MMP-9 (Figure 1d) were not detected immunohistochemically in lens epithelia cells preoperation.

\section{Lens capsule bag culture}

The capsule bags in culture media retained circular shape. Bags were full of media. The first signs of growth of lens epithelia cells at the equator were observed at day 4 (Figure 2). Then, lens epithelia cells migrated to the centre of the previously cell-free posterior capsule gradually (Figure 3). These cells ultimately covered the entire posterior capsule forming a confluent monolayer at day 20. Capsular bags remained largely wrinkle free until a complete cell cover of the posterior capsule had 

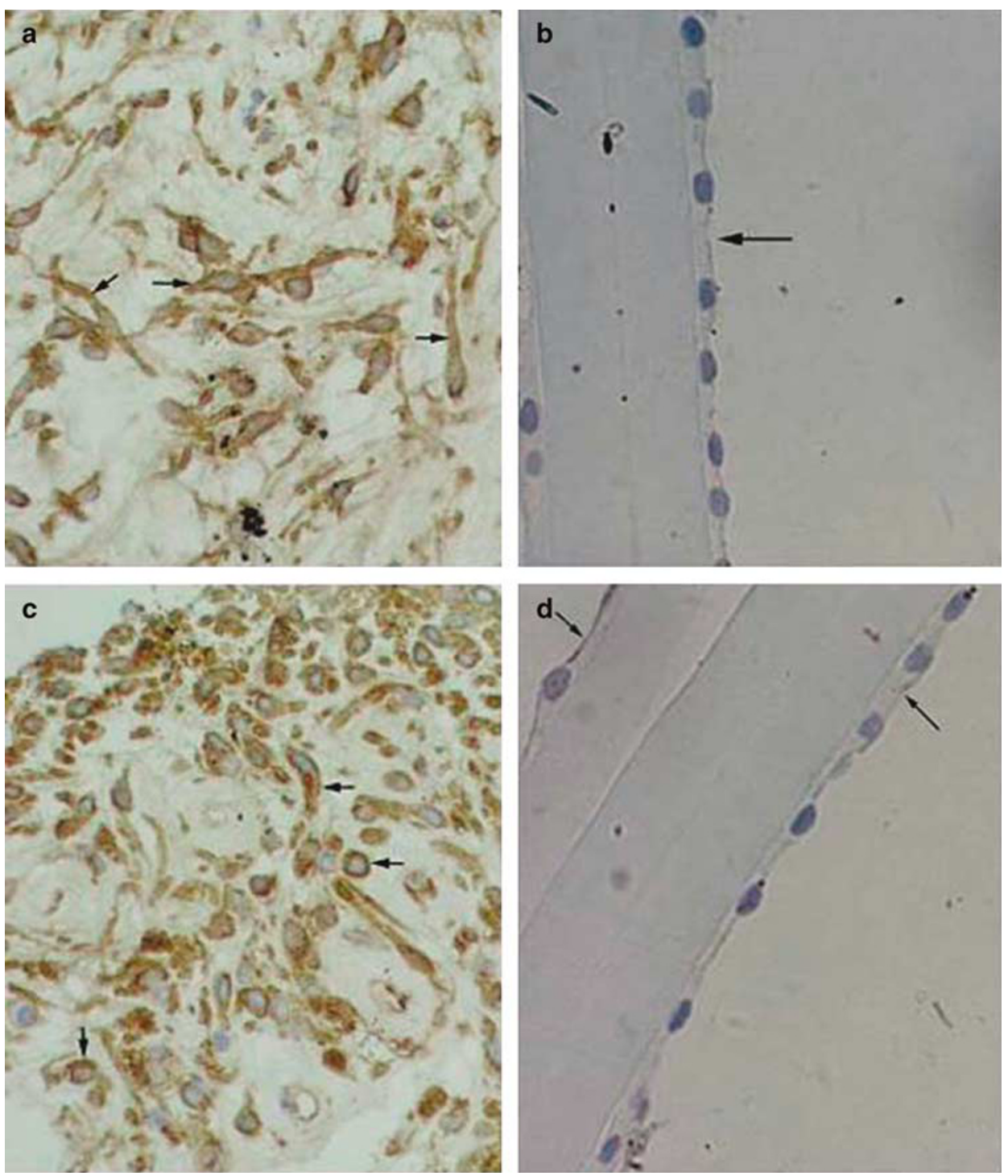

Figure 1 MMP-2 and -9 immunohistochemistry staining of human ciliary smooth muscle cells and human lens epithelial cells observed by light microscopy. Ciliary smooth muscle cells were immunostained with monoclonal antibodies directed against human MMP-2 (a) and MMP-9 (b). MMP-2 (c) and MMP-9 (d) were not detected by immunohistochemistry in human lens epithelial cells on the anterior capsulorhexis preoperation. Magnification $=\times 400$.

taken place. After this, the anterior capsule gradually adhered onto the posterior capsule partly and wrinkles began to develop at the centre of the posterior capsule (Figure 4). Capsule wrinkling and folds became increasingly apparent as time progressed.

\section{MMP production of media of cultured lens epithelia cells}

Total MMP-2 and -9 protein production was detected in samples obtained from capsular bag preparations at days 2, 10, 20, and 30, respectively. Total MMP-2 and -9 protein production increased with time with maximum levels reached on day 30 (Figure 5a and b).

\section{Discussion}

In the present study, for the first time, we observed the different expression of MMP-2 and -9 in lens epithelia cells between pre- and postoperation of sham cataract with the same donor human lens capsule in vitro. MMP-2 and -9 of human lens epithelia cells were showed to be upregulated following cataract operation, which was in agreement with other investigators. ${ }^{6,7}$

The requirement of MMP activity for the penetration and movement of several cell types through the ECM has been identified, ${ }^{2}$ which suggests that movement of cells through ECM may involve MMPs. The development of 


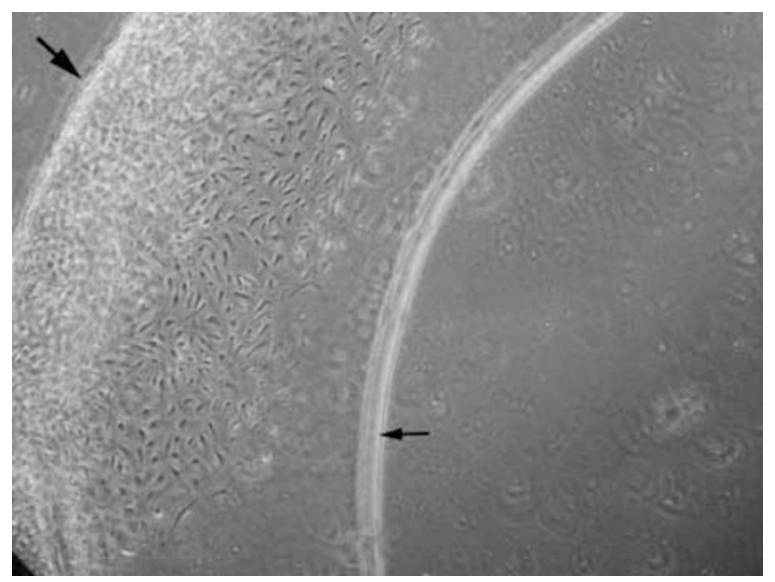

Figure 2 Phase-contrast micrograph of a human lens capsular bag cultured at day 4 . The capsule bag retained circular shape in culture media and was full of media. The first sign of growth of lens epithelia cells at the equator (thick arrow) was observed at day 4 . The edge of anterior capsulorhexis (thin arrow) was showed. Magnification $=\times 60$.

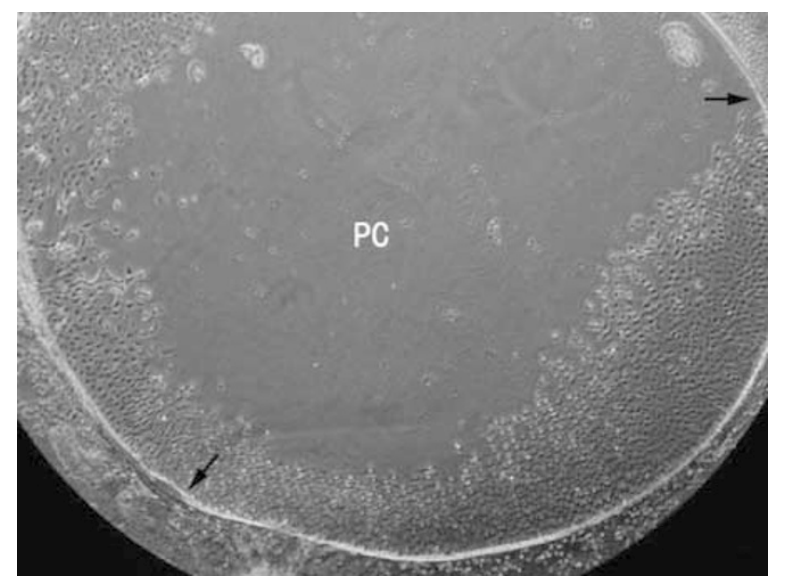

Figure 3 Phase-contrast micrograph of a human capsular bag cultured for 10 days. Lens epithelia cells were seen progressing toward the centre of the previously cell-free posterior capsule (PC). The edge of anterior capsulorhexis (black arrow) was showed. Magnification $=\times 40$.

PCO results from residual lens epithelia cell migration and proliferation. MMPs, therefore, deserve further attention and study to elucidate its importance in $\mathrm{PCO}$ formation.

Our data showed that the first signs of growth of lens epithelia cells at the equator were observed at day 4 . Then, lens epithelia cells migrate to the centre of the posterior capsule gradually. These cells ultimately covered the entire posterior capsule forming a confluent monolayer at day 20 following cataract surgery in serum-free culture medium and MMPs were upregulated at the same time. Total MMP-2 and -9 protein production increased with time with maximum levels reached on

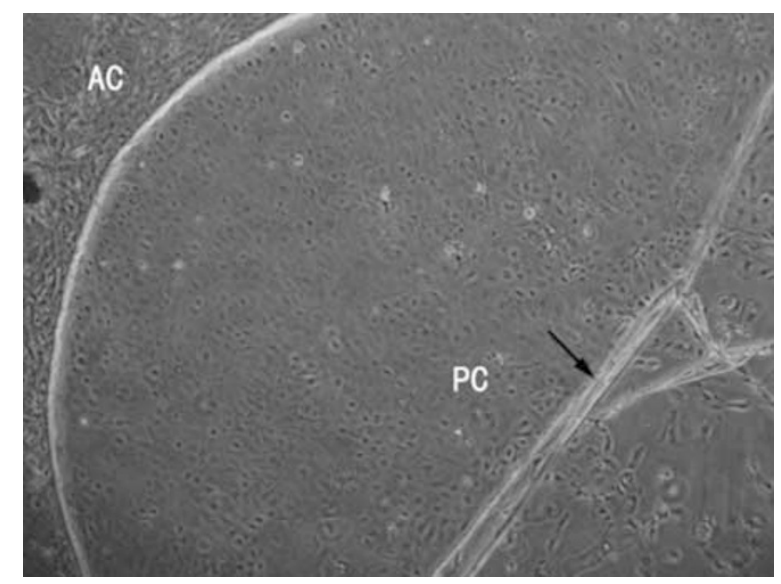

Figure 4 Phase-contrast micrograph of a human capsular bag cultured at day 30 . The capsule retained almost circular shape. Lens epithelia cells had formed a confluent layer on posterior capsule (PC) and the anterior capsule (AC) had a homogeneous appearance. Big wrinkles (black arrow) were noted at the centre of posterior capsule. The anterior capsule and the wrinkles were in focus at this level. Magnification $=\times 60$.

day 30. Therefore, MMPs probably improve the lens epithelia cells to migrate onto the posterior capsule. These cells then induce matrix modification such as wrinkling and cell aggregation, which results in light scatter and significant visual impairment.

MMPs require factors to stimulate expression in lens epithelia cells. ${ }^{8}$ The regulation of this induction could include matrix components and growth factors. The interaction between the cells and their ECM is now accepted as an important element in the regulation and function of all cell types. Lens epithelia cells survive and grow in capsule bag in serum-free media for more than 100 days. ${ }^{9}$ If lens epithelia cells detached from the capsule and cultured on plastic, they are unable to behave in the same manner. ${ }^{10}$ Moreover, MMPs of culturing chick lens annular pad (CLAP) cells was not detected using zymography on individual components of capsule matrix such as laminin, fibronectin, and collagen type IV. ${ }^{8}$ Although these individual matrix components are important in cell regulation, they do not truly reflect the full complement and structural organization of the lens capsule. In this study, we examined human lens epithelia cells attached to their natural matrix. In the case of closed lens capsule bags, mitotic rate of the lens epithelia cells was low due to contact inhibition. MMPs could not be detected by immunohistochemical method preoperation. However, total MMP-2 and -9 protein production in serum-free media increased following cataract surgery and increased with time. It would therefore appear that lens epithelia cells are capable of synthesizing MMP-2 and -9 by autocrine mechanisms, but seeming require stimuli to 

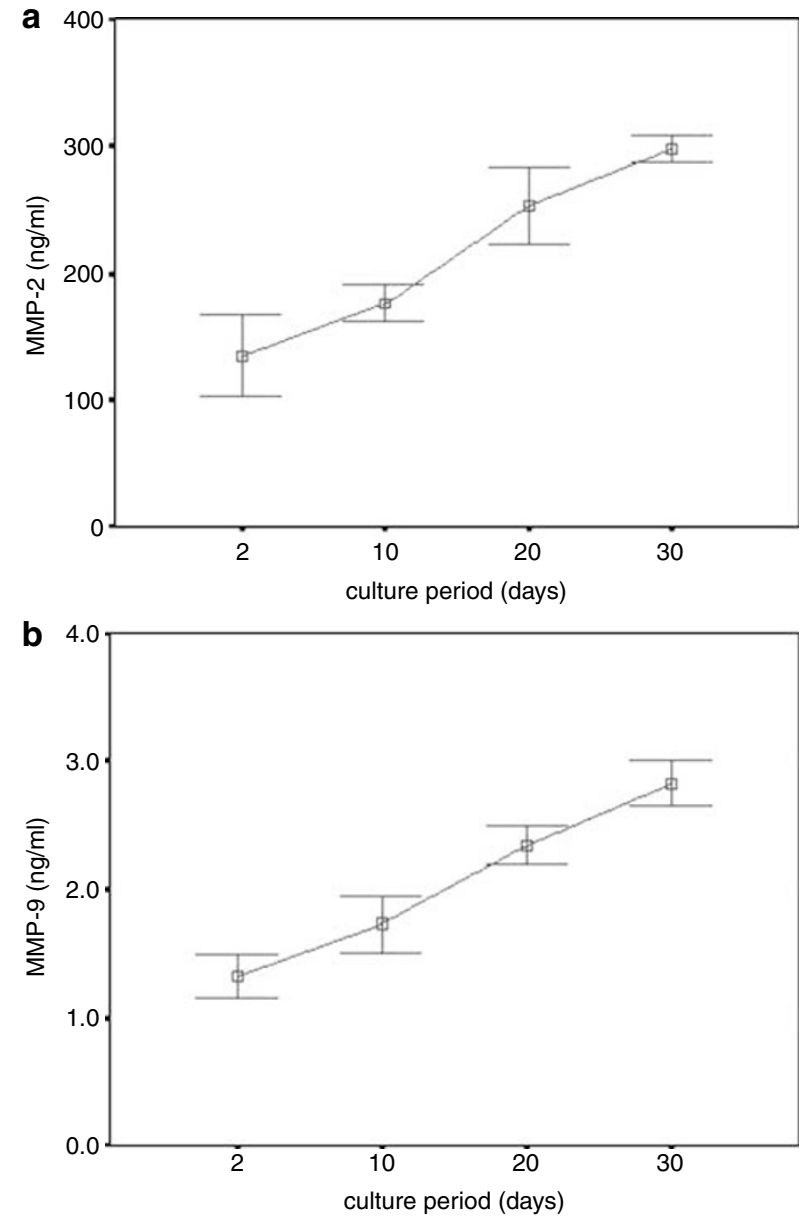

Figure 5 Total MMP-2 (a) and MMP-9 (b) protein production collected in the culture media of lens capsular bags quantified by ELISA. Both levers were increased with time with maximum levels reached on day 30. Error bars represent a 95\% confidence interval.

induce expression, such as cataract surgery. Damage to the epithelium would induce wound healing responses and was likely to involve upregulation of TGF- $\beta^{11}$ which in turn could induced synthesis of MMP-2 and -9. ${ }^{7}$ Richiert and Ireland ${ }^{8}$ found that TGF- $\beta$-induced expression of MMPs when added to CLAP cells cultured in serum-free medium, whereas controls did not express any activity. In this study, like Wormstone et al's $^{7}$ results, MMP-2 and -9 were detected by day 2 when no sign of growth of lens epithelia cells at the equator was observed, which means that cataract operation stimulated residual lens epithelia cells at the equator and under the anterior lens capsule to secrete MMP-2 and -9 . MMPs improve lens epithelia cells at the equator to migrate onto the previously cell-free posterior capsule.

The data in this study showed that more MMP-2 was produced than MMP-9 in culture media of human lens capsule bags. The observation was in agreement with
Wormstone et $a l^{7}$ who also found that there was a high lever of MMP-2 and a detected lever of MMP-9 within 2 days of serum-free culture media of human capsule bags using gelatin zymography. The result indicates that MMP-2 probably plays a main role in human lens epithelia cell migration.

In the present system, capsule bags were maintained in a serum-free medium. In this study, it was not feasible to utilize serum, as it possesses high endogenous amounts of MMPs and could have contained some growth factors, such as TGF- $\beta$, which could increase production of MMPs. ${ }^{7}$ In addition, while serum factors can stimulate lens epithelia cell growth they are not essential for lens epithelia cell growth to take place. ${ }^{9}$

In our study, no pin was used to fix the capsule bag, which is different from the technique of Liu et al. ${ }^{4}$ The capsule bag in the media was opened by shaking the culture plate gently as soon as the bag was dissected free of the zonules and transferred into culture media. The capsule bag in culture media retained circular shape and was full of media all the time. The movement of the bag in the media did not influence the migration of lens epithelia cells in the bag.

We did not examine opacified lenses as all lenses of donated eyes were not cataractous.

Using the in vitro capsular bag model, we have shown that MMPs probably play an important role in development of PCO. Therefore, MMP inhibitors could be of potential therapeutic benefit to $\mathrm{PCO}$ after cataract surgery in the future.

\section{Acknowledgements}

We thank Jing Liu of the Tongren Eye Bank for her invaluable work.

\section{References}

1 Wong TT, Sethi C, Daniels JT. Matrix metalloproteinases in disease and repair processes in the anterior segment. Surv Ophthalmol 2002; 47: 239-256.

2 Ye HQ, Azar DT. Expression of gelatinase A and B and TIMPs 1 and 2 during corneal wound healing. Invest Ophthalmol Vis Sci 1998; 39: 913-921.

3 Mackay AR, Gomez DE, Cottam DW. Identification of the 72-kDa ( MMP-2) and 92-kDa ( MMP-9) gelatinase/type IV collagenase in preparations of laminin and Matrigel. Biotechniques 1993; 15: 1048-1051.

4 Liu CS, Wormstone IM, Duncan G, Marantonio JM, Webb SF, Davies PD. A study of human lens cell growth in vitro. A model for posterior capsule opacification. Invest Ophthalmol Vis Sci 1996; 37: 906-914.

5 Weinreb RN, Kashiwagi K, Kashiwagi F, Tsukahara S, Lindsey JD. Prostaglandins increase matrix mentalloproteinase release from human ciliary smooth muscle cells. Invest Ophthalmol Vis Sci 1997; 38: 2772-2780. 
6 Tamiya S, Wormstone IM, Marcantonio JM, Gavrilovic J, Duncan G. Induction of matrix metallo proteinases 2 and 9 following stress to the lens. Exp Eye Res 2000; 71(6): 591-597.

7 Wormstone IM, Tamiya S, Anderson I, Duncan G. TGF- $\beta 2$-induced matrix modification and cell transdifferentiation in the human lens capsular bag. Invest Ophthalmol Vis Sci 2002; 43: 2301-2308.

8 Richiert DM, Ireland ME. Matrix metalloproteinase secretion is stimulated by TGF-b in cultured lens epithelial cells. Curr Eye Res 1999; 19: 269-275.
9 Wormstone IM, Del Rio-Tsonis K, McMahon G, Tamiya S, Davies P, Marcantonio J et al. FGF: an autocrine regulator of human lens cell growth independent of added stimuli. Invest Ophthalmol Vis Sci 2001; 42: 1305-1311.

10 Bermbach G, Mayer U, Naumann GO. Human lens epithelial cells in tissue culture. Exp Eye Res 1991; 52: 113-119.

11 Ohta K, Yamagami S, Taylor AW. IL-6 antagonizes TGF-beta and abolishes immune privilege in eyes with endotoxin-induced uveitis. Invest Ophthalmol Vis Sci 2000; 41: 2591-2599. 